\title{
Proposta de nomenclatura de cor para pesquisa de impressos históricos
}

\author{
Color nomenclature proposal for historical prints research
}

\author{
BARROS, Helena de; Doutoranda; ESDI/ UERJ \\ helenbar@helenbar.com \\ LESSA, Washington Dias; DSc, ESDI/UERJ \\ washington.lessa@gmail.com \\ LIMA, Edna Cunha; DSc; PUC-RJ \\ ednacunhalima@gmail.com
}

\section{Resumo}

Este artigo se insere na metodologia de pesquisa da investigação de uma técnica de impressão histórica, a cromolitografia, e sua aplicação comercial no Brasil entre 1876 e 1919. Ao longo da pesquisa, verificou-se a necessidade da adoção de uma nomenclatura de cor especifica, que atendesse aos seus objetivos: identificação, caracterização e diferenciação das cores. Foram pesquisadas nomenclaturas de cor contemporâneas ao estudo (meados do século XIX e início do século XX), até a adoção de um sistema descritivo, lógico e compreensivo, baseado no Sistema de Cor de Munsell, na Linguagem de Cor Universal e na terminologia proposta pela ISCC-NBS. A proposta inclui a confecção de uma escala de referência cromática, exemplificando seu uso prático nesta pesquisa.

Palavras Chave: cor, nomenclatura, linguagem de cor universal.

\section{Abstract}

This paper fits in the research methodology of the investigation of a historical printing technique, chromolithography, and its commercial application in Brazil between 1876 and 1919. In the course of the research the necessity of adoption of a specific color nomenclature was identified, attending its objectives: color identification, characterization and differentiation. Color nomenclatures contemporary to the study period (mid XIX century to early XX century) were researched, until the adoption of a descriptive, logical and comprehensive system based on Munsell Color System, Universal Color Language and on the terminology proposed by ISCC-NBS. This proposal includes the execution of a chromatic reference scale, exemplifying its practical use in this research.

Keywords: color, nomenclature, universal color language. 


\section{A necessidade de estabelecer uma nomenclatura de cor específica}

O tema abordado neste artigo se insere numa pesquisa que investiga uma técnica de impressão histórica que vigorou entre meados do século XIX e a primeira metade do século XX, a cromolitografia ${ }^{1}$, e sua aplicação comercial no Brasil. O corpus de pesquisa constituiu-se a partir das coleções de rótulos de produtos brasileiros impressos pela técnica cromolitográfica, resultando na seleção de 100 amostras em acervos de instituições públicas da cidade do Rio de Janeiro - Arquivo Nacional e Biblioteca Nacional -, produzidas entre 1876 e 1919.

A aproximação do tema nomenclatura de cor surgiu no decorrer da pesquisa quando, na aproximação técnica, verificou-se a necessidade de identificar, nomear e diferenciar as cores de seleção observadas nas amostras do corpus de estudo. Nesta proposta de investigação, as cores são identificadas através de observação microscópica, revelando por pequenos desencontros de registro, os matizes componentes das amostras impressas. Apesar de não haver uma necessidade de identificação precisa, julgou-se relevante apontar os distintos conjuntos de cores utilizadas em cada rótulo - na cromolitografia, uma cor com aparência de cor sólida a olho nu, pode ser formada por 5 ou mais tintas diferentes sobrepostas e a pesquisa trata justamente da análise desta complexa proposta de construção cromática. As tintas eram desenvolvidas caso a caso, obtidas por misturas, de acordo com a necessidade de representação de cada rótulo, sem uma nomeação específica.

$\mathrm{Na}$ única prova de cor impressa encontrada na coleção de rótulos da Biblioteca Nacional (BN), o rótulo da Água Ardente Superior (Figura 1). Verifica-se que a nomeação das cores praticada pelos cromistas visava apenas criar uma distinção mínima das tintas operantes (ou cores de seleção) utilizadas na impressão, sem nenhuma pretensão de caracterizá-las. Para melhor observação, destacamos e rebatemos a anotação que identifica as 8 cores utilizadas nesta composição. Por se tratar de uma técnica de impressão direta, a cromolitografia exigia que a matriz fosse gravada de forma espelhada, para adquirir o sentido inverso na transferência da tinta para o papel - como a anotação de cor era uma observação interna entre cromistas impressores, descartada no produto final, dispensava a tarefa mais laboriosa de gravar o texto invertido, o que justifica a ocorrência impressa de textos nos dois sentidos de leitura (da esquerda para direita e da direita para esquerda). Aqui é possível comprovar a participação e expertise do cromista ${ }^{2}$ estrangeiro, já que as cores são identificadas no idioma alemão: gold (dourado), tiefe (profundidade), gelb (amarelo), blau 1 (azul 1), ton (argila), grau (cinza), rot (vermelho), blau 2 (azul 2) e rosa (rosa). Como se pode notar, a diferenciação entre as duas tonalidades de azul, se dá apenas pela sufixação de números, para indicar o uso de mais de um azul, e exceto o termos tiefe (profundidade, utilizado para indicar as áreas de sombra), e ton (argila, utilizado para indicar uma tonalidade de amarelo rosado), a nomenclatura de cor restringe-se aos termos mais básicos e essenciais. O pesquisador

\footnotetext{
${ }^{1}$ Segundo Mazio, a cromolitografia é uma impressão litográfica colorida onde a imagem é composta por ao menos três cores, cada uma aplicada no impresso por uma pedra diferente. Ao contrário da litografia entintada, onde a segunda e a terceira cor distribuem matizes sobre a primeira impressão, as cores de uma cromo constituem a figura em si. A cromolitografia é portanto uma técnica muito complexa que requer registro perfeito e um sofisticado entendimento da cor." (MARZIO, 1979, p. 9, tradução nossa).
}

\footnotetext{
${ }^{2}$ Profissional responsável pela concepção de cor na cromolitografia.
} 
inglês Michael Twyman comenta a respeito das particularidades e variações de nomeação de cor usadas por alguns impressores: "as cores eram graduadas de I-III de acordo com sua saturação." (TWYMAN, 2013, p.584)

Figura 1 - Rótulo Superior Aguardente Portuguesa, Bagaceira e Vinho Verde Flôr de Amarante, Soares de Azevedo e Co., 21,8 x 17,5cm, 1917 e detalhe ampliado e rebatido da barra de cores de impressão.
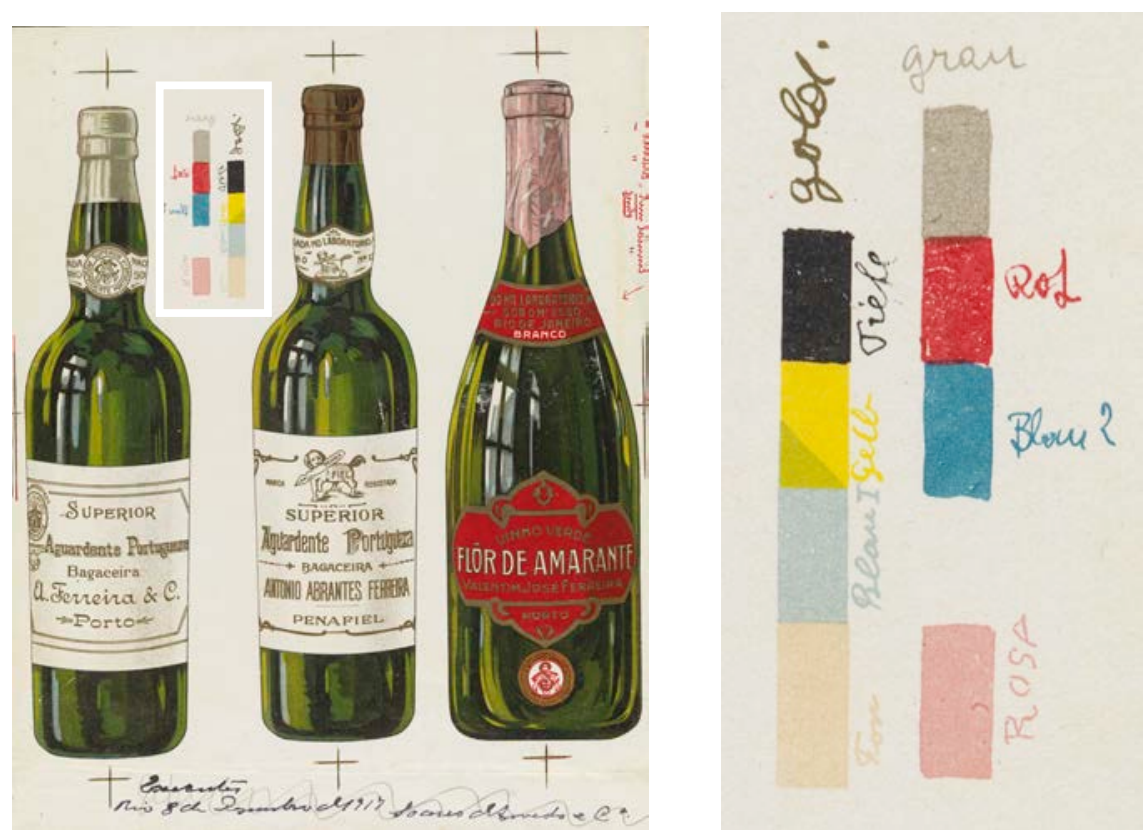

Fonte: Acervo da Fundação Biblioteca Nacional - Brasil

Já que no próprio linguajar técnico da cromolitografia, as cores não eram nomeadas ou identificadas com precisão, por que então percebemos a necessidade de uma nomenclatura mais especifica nesta pesquisa? A resposta se justifica no cruzamento de alguns fatores:

1. Não tratamos de uma única amostra, mas de um conjunto abrangente (constituído por 100 rótulos). Assim, nas análises comparativas, se faz necessário identificar, por exemplo, se um determinado azul é mais utilizado do que outro, a frequência de tonalidades semelhantes como recurso recorrente, ou a necessidade de matizes mais específicos de acordo com o efeito cromático desejado. A identificação desses matizes é um elemento essencial para compreensão da operação de construção de cor, suas bases e efeitos.

2. Uma nomenclatura de cor mais específica pode facilitar o discurso de composição cromática, numa compreensão mais intuitiva das misturas e obtenção de tonalidades, em oposição a um discurso excessivamente árido e hermético, se associado apenas a valores numéricos ou códigos de identificação de cor.

3. É proveitoso desenvolver e tornar mais preciso o vocabulário técnico, trazendo uma aproximação que não pareça avessa ao contexto histórico das amostras. A nomenclatura de cor passou a ser mais estudada e desenvolvida ao longo do século XIX e início do século XX, sendo um conhecimento de apoio que enriquece o trabalho em seu contexto histórico.

4. É necessário ainda estabelecer correspondências com referências de cor atuais, 
que possam situar o leitor num contexto mais preciso e confiável do que a tecnologia de impressão de artigos e teses (que não oferece precisão cromática). Nesse sentido, independente da nomenclatura escolhida, a tabela de cor adotada irá oferecer correspondências de cor aproximadas com alguns sistemas de identificação de cor mais comumente utilizados por designers na atualidade: o sistema aditivo de feixes luminosos RGB (verde, vermelho e azul) em sua codificação hexadecimal, e o sistema subtrativo de tintas translúcidas baseado em CMYK (ciano, magenta, amarelo e preto).

Apesar de não haver aqui um rigor científico preciso na medição de cor, especialmente pelas condições de execução da pesquisa ${ }^{3}$, a nomenclatura de cor neste trabalho indica o desejo de aplicar um critério unificado e suficientemente matizado de aproximação. Tal critério deve ser capaz de dar suporte à compreensão da natureza lógica da construção de cor e sua estrutura de funcionamento. Semelhante a uma operação matemática, quando nomeamos adequadamente suas bases componentes, podemos compreender melhor suas operações (gradação tonal, superposição, somatória de percentagens, etc.) e a diferenciação de resultados. O discurso relativo às cores está diretamente vinculado aos termos que se adotam na sua verbalização, à compreensão do vocabulário e à sua correspondência no mundo real, ou seja, do léxico. Logo, para que o discurso seja assimilado como lógico e compreensível, é imprescindível a adoção de uma linguagem pertinente, precisa e padronizada. "Imagens mentais claras formam um discurso claro. Pensamentos vagos encontram termos vagos." (MUNSELL, 1907, p.13, tradução nossa)

\subsection{Sistemas e propostas de nomenclatura de cor}

A primeira opção cogitada para nomenclatura de cor foi adotar o mesmo sistema utilizado atualmente no meio gráfico para a especificação de cores sólidas: o Pantone Matching System, da empresa norte americana Pantone Inc., criada em 1963. Este sistema ofereceria uma gama de cores bastante diversificada com 1044 tintas, baseadas na mistura material de 14 tintas básicas. Porém, apenas as tintas básicas recebem uma nomeação textual (ex.: Yelow, Warm Red, Reflex Blue, etc.) as demais tintas são identificadas por código numérico (ex.: 178, 5235, etc.). Assim a Pantone incorreria nos itens 2 e 3 acima apontados: o uso de números tornaria o discurso árido, de difícil compreensão, implicando na necessidade do leitor consultar a escala para a identificação dos matizes, e principalmente, pareceu-nos anacrônico e incoerente usar um sistema contemporâneo tão específico para designar tintas de um período já tão distante no tempo - a pesquisa se situa entre 1876 e 1919, encerrando-se mais de quatro décadas antes do estabelecimento da Pantone como referência gráfica.

Assim, iniciou-se uma pesquisa histórica sobre nomenclatura de cor que fosse mais próxima do período histórico do nosso recorte (meados do século XIX e inicio do século $X X$ ). Esta pesquisa nos levou ao conhecimento do primeiro esforço científico de padronização de nomes das cores: o Werner's Nomenclature of Colours (Figura 2), editado no Reino Unido em 1821 pelo pintor Patrick Syme, baseando-se no trabalho anterior do mineralogista alemão Abraham Gottlob Werner (1749-1817). O livro se autoproclama como o primeiro a oferecer uma proposta unificada de nomenclatura de cor,

\footnotetext{
${ }^{3}$ Entre elas, a falta de iluminação adequada nas salas de consulta das instituições, o desgaste e desbotamento das tintas em exemplares históricos, a dificuldade e imprecisão na comparação de elementos microscópicos com amostras impressas, etc.
} 
com correspondências na zoologia, botânica, química, mineralogia, anatomia e artes.

\begin{abstract}
Uma nomenclatura das cores, com exemplos apropriados de cor nas diferentes tintas, como um padrão genérico para ser usado na descrição de qualquer objeto, há muito tem sido desejado nas artes e ciências. Uma coisa tão obviamente útil na descrição de objetos da história natural e das artes, onde a cor é um objeto indispensavelmente necessário, já deveria há muito ter sido alcançado. (SYME, 1821, pág. 5-6, tradução nossa)
\end{abstract}

Empreendimento louvável, a obra apresenta 110 amostras de cor nomeadas de acordo com tintas ou pigmentos adotados na pintura, em tabelas com a contextualização de objetos conhecidos do reino animal, vegetal e mineral, compreendendo as cores ou tintas mais comuns na natureza. Situado num período anterior ao surgimento da cromolitografia, o que poderia favorecer sua adesão, a terminologia proposta é heterogênea, variando entre o técnico, artístico e o referencial como por exemplo "Púrpura Pálido Enegrecido", "Púrpura Lilás Azulado", "Azul Índigo", "Azul Imperial", "Vermelho Carmim" ou "Vermelho Sangue Venoso". O catálogo completo está disponível online em versão digital, porém, a maior critica da época foi a falta de permanência das amostras, que desbotavam rapidamente, oferecendo assim, pouca acuidade como fonte de referencia.

Figura 2 - Werner's Nomenclature of colours, Patrick Syme, 1821

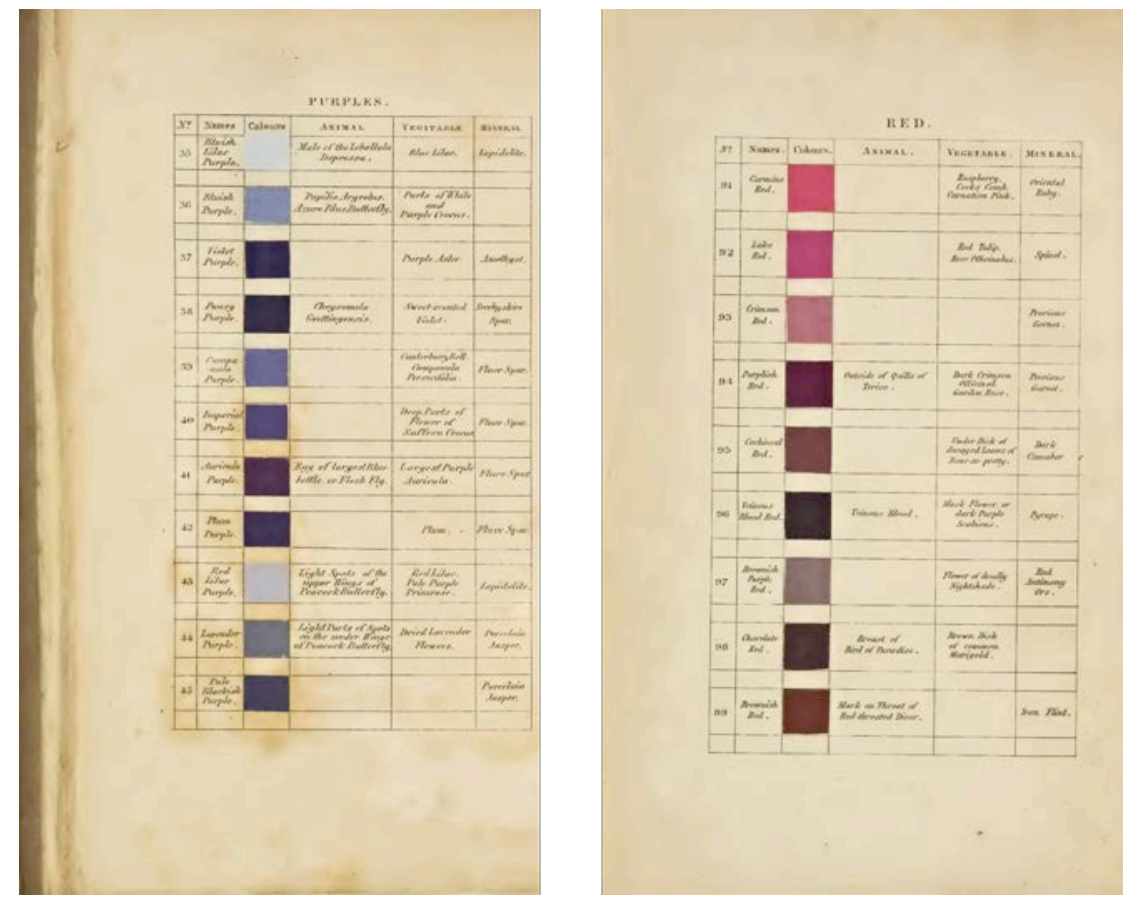

Fonte: SYME, 1821.

A próxima publicação relevante destinada à nomenclatura de cor, viria a ser do ornitólogo americano Robert Ridgway, A Nomenclature of Colors for Naturalists and Compendium of Useful Knowledge for Onritologists, publicada em Boston no ano de 1886. Ridgway demonstra uma preocupação de ordem normativa com a nomenclatura de cor, estabelecendo um vocabulário comparativo em vários idiomas (inglês, latim, alemão, francês, espanhol, italiano e dinamarquês) e apresentando 186 amostras de cores estáveis, pintadas em tinta a óleo, desenvolvidas pela consagrada fabricante de tintas para pintura Windsor and Newton. 
Sua obra foi aprimorada posteriormente com uma segunda publicação, Color Standards and Color Nomenclature, de 1912 (Figura 3), desta vez nomeando 1.115 cores, ilustradas com amostras impressas separadamente e coladas em 53 pranchas, considerada até hoje uma referência respeitada. Ridway teve importantes colaboradores nesta segunda edição, entre eles o renomado físico Ogden Nicholas Rood, responsável por avanços na teoria da cor e chegou a nomear uma das amostras como Azul Rood (Rood's Blue) em sua homenagem. A data de ambas as publicações coincide com o período de amostragem do nosso corpus de estudo, foram digitalizadas e estão disponíveis online na íntegra. Porém, a adoção de alguns termos excessivamente personalizados (como o citado acima), fantasiosos como o "Vermelho Brasil" (Brazil Red) ou até poéticos, como por exemplo "Vermelho Sangue de Dragão" (Dragon's Blood Red), da publicação anterior, fazem com que estas obras se tornem pouco úteis sob o aspecto descritivo de caracterização da cor.

Figura 3 - Color Standards and Color Nomenclature, Robert Ridway, 1912, com 1.115 amostras de cores impressas e coladas separadamente.
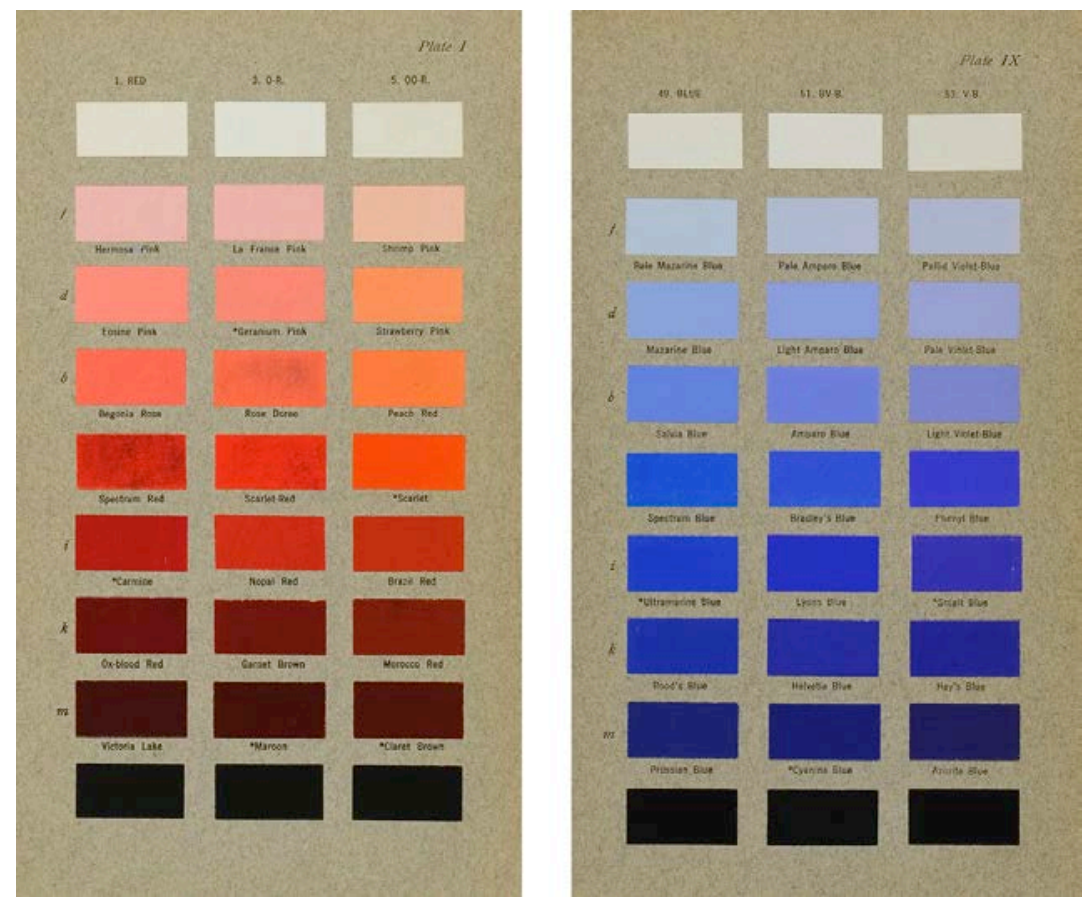

Fonte: RIDWAY, 1912.

Munsell comenta sobre a ineficiência dos termos da época:

Onde poderia ser encontrado mais prazeroso clamor por uma forma racional de descrever a cor? Ele quer "um amarelo topázio e um vermelho que não é Turco, nem Romano, nem Indiano", mas que "parece ter parte dos dois últimos e ainda assim não ser nenhum deles". Como o ápice do clímax, ele exige "amostras do tom exato". Desse modo, um dos escritores mais claros e potentes da língua inglesa (Robert Louis Stevenson) se vê incapaz de descrever a cor que deseja. E por quê? Simplesmente porque a linguagem popular não estabelece de forma clara nenhuma das três qualidades constitutivas de cada cor, que precisam ser conhecidas antes que se tenha a expectativa de transmitir concepções de cor a alguém. A incongruente e bizarra natureza dos nomes de cor atuais transparecem a qualquer pessoa de bom senso. Termos que expressam ideias diferentes para 
pessoas diferentes e que fracassam completamente na tentativa de definir as cores. Convites ao equívoco e ao desapontamento. Não são termos apenas imprecisos, mas inapropriados. (MUNSELL, 1905: p.169, tradução nossa)

Twyman (2013) comenta que chegou a considerar se referir as cores na cromolitografia por meio de algum sistema de cor reconhecido. No aspecto menos cientifico, ele recomendaria o dicionário usado por filatelistas, $A$ colour dictionary giving two hundred names of colours. Specially prepared for stamp collectors, Warhurst, c. 1908. A nomenclatura de cor neste título adota nomes de pigmentos mais comuns na pintura, como por exemplo carmim, escarlate e vermelhão para diferenciar vermelhos (Figura 4). Julgou-se que este tipo de nomenclatura, apesar de ser mais próximo do universo dos cromistas, não seria muito claro para o universo dos designers de hoje, e continuou-se a buscar uma nomenclatura mais descritiva.

Figura 4 - A colour dictionary giving two hundred names of colours. Specially prepared for stamp collectors, 2nd edn., B.W. Warhurst, 1905.

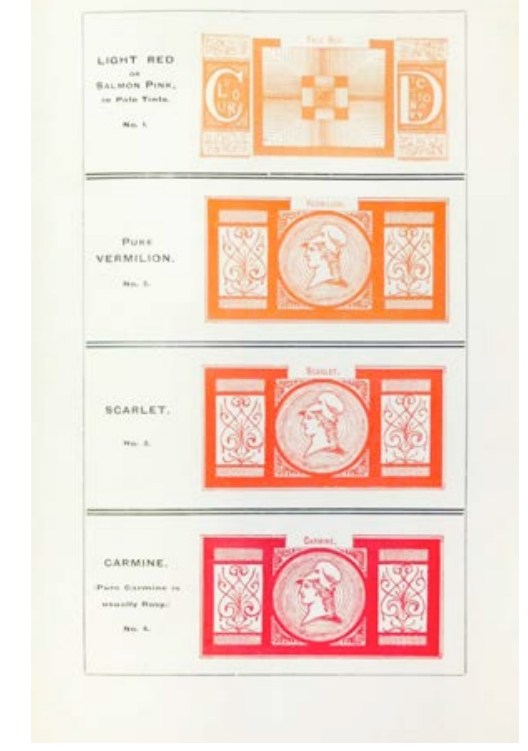

Fonte: WARHURST, 1905.

O trabalho mais significativo para o nosso intuito foi o modelo criado pelo artista plástico e educador Albert Henry Munsell (1858-1918), o Munsell Color System, proposto inicialmente em 1898. A esfera de Munsell teve seus valores descritos em 1905, foi publicada como um atlas de cor em 1915 e refinada em 1929, com a publicação do Munsell Book of Color. Este foi revisado em 1943 pela Optical Society of America, a fim de se aproximar mais precisamente do desejo de Munsell de um sistema de cor funcional e perceptualmente balanceado (Figura 5a), sofrendo atualizações mais significativas em 1970 e 1993. O Munsell Book of Color identificou inicialmente 1.722 cores, ilustradas com amostras impressas em 40 pranchas. É um sistema de ordenação de cores perceptualmente uniforme que possibilita um arranjo num espaço tridimensional (Figura $5 b)$. As cores são organizadas a partir dos conceitos matiz, croma (ou saturação) e valor (ou luminosidade) numa base lógica e descritiva, com referenciais numéricos, associados aos principais matizes de acordo com sua sequencia no disco cromático. Munsell estabeleceu 5 eixos principais: R (Red, vermelho), Y (Yellow, amarelo), G (Green, verde), 
$\mathrm{B}$ (Blue, azul) e $\mathrm{P}$ (Purple, púrpura). A combinação das duas iniciais indicam os matizes intermediários: RY (Red-Yellow), vermelho-amarelo indica o laranja, YR (Yellow-Red) um amarelo alaranjado, YG (Yellow-Green) o amarelo esverdeado e assim por diante, perfazendo 10 eixos principais. Dessa forma, o matiz (hue) é representado por angulações, ao redor do sólido tridimensional.

Figura 5 - a) Disco cromático de Munsell; b) Representação do espaço de cor tridimensional na árvore de cor de Munsell e c) Representação tridimensional de Matiz, Valor e Croma segundo Munsell.

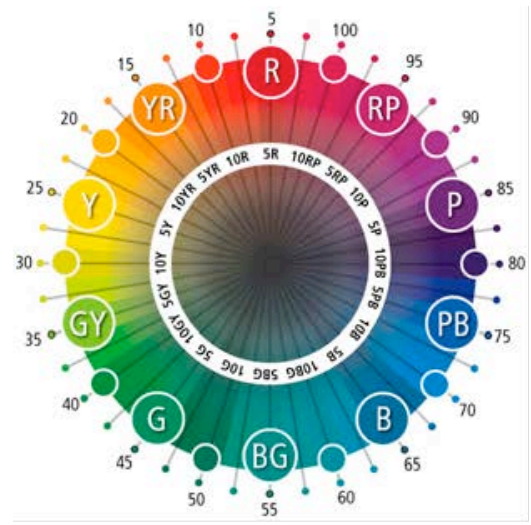

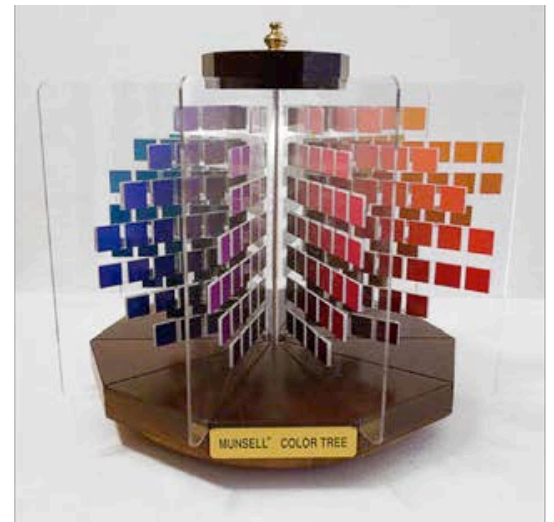

Fonte: www.munsell.com

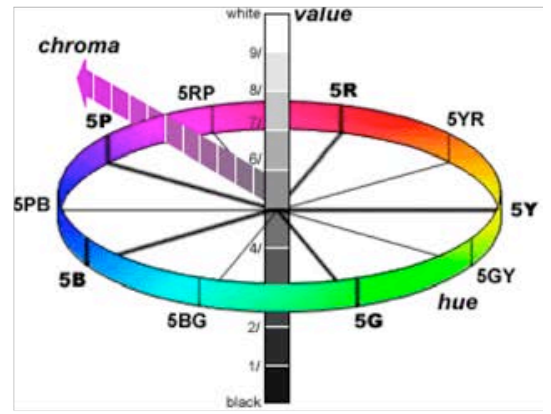

O círculo de Munsell é dividido em 10 setores angulares, cada um com angulo de $36^{\circ}$, cada setor sendo dividido em 10 subsetores, variando portanto de 1 a $10^{\circ}$ para cada matiz. O parâmetro de valor (ou luminosidade) se estabelece no eixo vertical, de baixo para cima, mensurado de 0 a 10 por valores Neutros (N1 a N10). O parâmetro de croma varia do centro ao exterior em cortes horizontais do sólido tridimensional (Figura $5 \mathrm{c}$ ). Inicialmente proposto por Munsell na variação de 1 a 8 , atualmente os catálogos chegam ao croma 18, mas não há limite finito arbitrado para este parâmetro. À medida que novos pigmentos se tornam disponíveis no mercado, a escala pode ser estendida (algumas cores fluorescentes, por exemplo, podem chegar ao croma 30) (munsel.com).

A especificação de cor se dá no formato H V/C (Hue Value/Chroma). Para um vermelho vívido, por exemplo, de matiz 5, valor 6 e croma 14, a notação completa é $5 R$ 6/14 (Figura 6), onde 5R (Red) é o código para um vermelho intermediário, 6 significando luminância mediana e a saturação 14 indicando um alto grau de pureza. A notação obedece uma escala variável correspondendo às diferentes extensões, de acordo com a natureza perceptual de saturação de cada matiz e da sua posição em relação à luminosidade. 
Figura 6 - Exemplo de posicionamento (notação 5R 6/14)

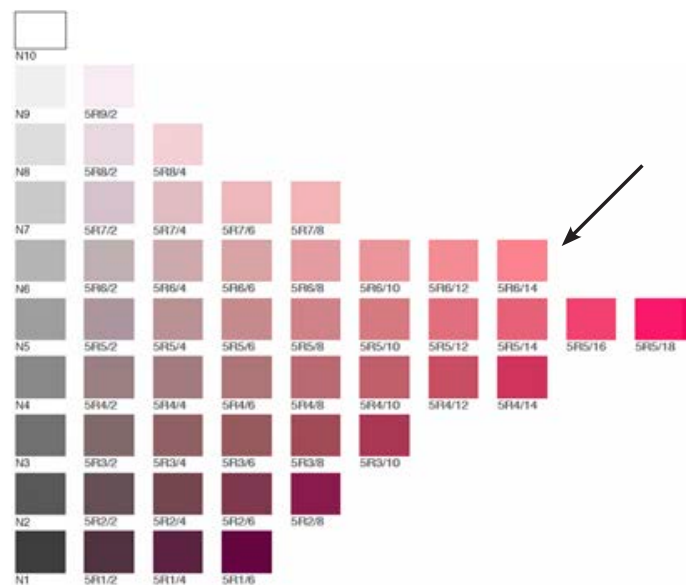

Fonte: http://print-color-charts.com/

Referência internacional definida pela ASTM (American Society of the International Association for Testing and Materials) na norma D1535, o Sistema de Munsell baseia-se em livros de amostras referenciais e é adotado atualmente por artistas, designers, cientistas, engenheiros e reguladores do governo, para especificar cores existentes ou desejadas em diversos campos de atuação como arqueologia (cores de artefatos), estudos médicos (referenciando tons de pele em doenças), geologia (para tonalidades de solo) e até hobbys como, por exemplo, para determinar a cor de aviões antigos.

O livro Color Universal Language Dictionary of Names (KELLY, 1976), publicado pelo Center for Building Technology do National Bureau of Standards norte americano, estabeleceu uma nomenclatura compatível com o Sistema de Munsell e um dicionário de 7.500 nomes de cores: o UCL, Universal Color Language. Compilação de todos os sistemas comerciais e métodos de designação de cor, o UCL tem os seguintes objetivos:

a) Ser apurado o suficiente para satisfazer o cientista (nível 6 e 5 - 5.000.000 e 100.000 cores);

b) Utilitário o suficiente para satisfazer o fabricante (nível 4 e 3 - 7.056 a 267 cores);

c) Simples o suficiente para ser entendido pelas pessoas comuns (nível, 1, 2 e 3 13, 29 e 267 cores).

Assim, temos:

1. O nível 1, com 13 cores: Red (vermelho), Orange (laranja), Yellow (amarelo), Yellow-green (verde amarelado), Green (verde), Blue (azul), Purple (púrpura), White (branco), Gray (cinza), Black (preto), Pink (rosa), Brown (marrom), e Olive (oliva). Note-se a adoção dos termos "laranja","rosa", "marrom" e "oliva", pela força do hábito no uso comum.

2. O nível 2, com 29 cores, acrescenta Reddish orange (laranja avermelhado), Orange-yellow (amarelo alaranjado), yellowish pink (rosa amarelado), brownish pink (rosa amarronzado), reddish brown (marrom avermelhado), brownish orange (laranja amarronzado), yellowish brown (marrom amarelado), olive-brown (marrom-oliva), greenish yellow (amarelo esverdeado), Olive-green (verde oliva), yellowish green (verde amarelado), bluish green (verde azulado), greenish blue (azul esverdeado), purplish blue 
(azul purpurado), violet (violeta), reddish purple (púrpura avermelhado), purplish pink (rosa purpurado) e purplish red (vermelho purpurado);

3. O nível 3 apresenta 267 cores nomeadas, a partir de variações básicas de luminosidade e saturação;

4. O nível 4 é o próprio Munsell Book of Color (1.722 cores);

5. O nível 5 são as cores de Munsell interpoladas e,

6. Finalmente, o nível 6 tem em torno de 5,000,000 de cores designadas pela CIE (Comission Internationale d'Eclairage), em valores matemáticos, sem nomes.

A nomenclatura do nível 3 foi designada entre 1933 e 1935 pela ISCC-NBS - InterSociety Color Council, ISCC / United States Department of Commerce's National Bureau of Standards, NBS - por meio do emprego de oito adjetivos modificadores de luminosidade e de saturação (pálido, claro, moderado, escuro, brilhante, forte, profundo e vívido) e o advérbio de intensidade "muito" (JUDD \& KELLY, 1939). Porém, de acordo com a notação de Munsell, os 267 termos não designam tons singulares, mas áreas com variação de ao menos 7 tonalidades distintas no espaço tridimensional. Com o objetivo de facilitar uma identificação genérica, a ISCC-NBS propôs uma equação matemática para calcular os valores intermediários destas tonalidades, resultando em valores centroides (centroids), que designam uma única cor mediana para cada termo (NICKERSON, 1940). Mais do que nomear as cores, acreditamos que a terminologia baseada no modelo de Munsell da ISCC-NBS (Tabela 13) estabelece a compreensão descritiva e o mapeamento de um sistema cromático lógico.

Figura 7 - Esquema compreensivo dos adjetivos modificadores de Luminosidade e Saturação de acordo com a ISCC-NBS.

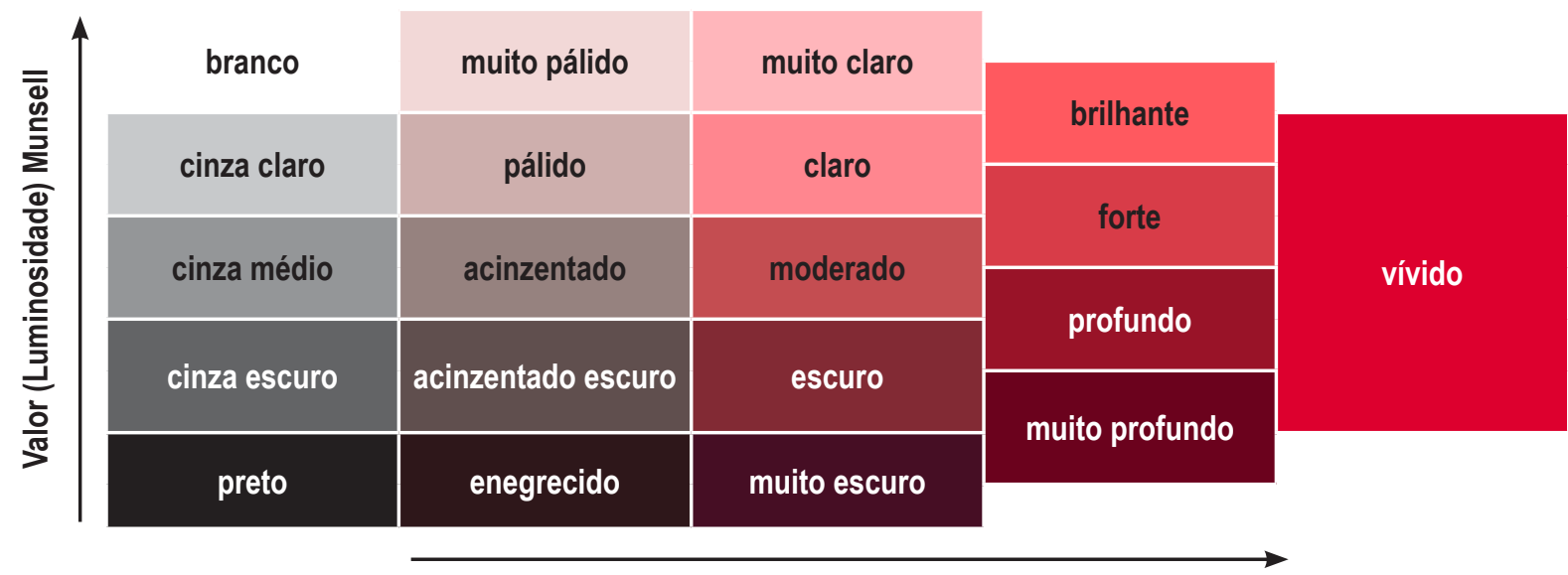

Croma (Saturação) Munsell

Fonte: a autora, elaborado a partir de KELLY \& JUDD, 1976, pág. A-16, tradução e colorização nossa.

Com o objetivo de nomenclatura padronizada, que desse conta da variedade de matizes presentes nas amostras, sem ser excessivamente extensa nem insuficiente, a nomenclatura do nível 3 proposta pela ISCC-NBS foi a que julgamos mais adequada para identificação de cor nesta tese. Os termos acompanhados das referências centroides na notação de Munsell, acrescidos da correspondência para a codificação de valores hexadecimais RGB (realizada por John C. Foster para um clube de colecionadores de selos norte americano) foram referenciados pela tabela disponibilizada no site 
http://tx4.us/nbs-iscc.htm (Copyright $@$ 2004-2006 Voluntocracy).

A tabela completa contempla a nomeação e representação de 267 cores. Foi acrescida a tradução (nossa) para os termos correspondentes em português e a transposição aproximada para valores em CMYK, calculada manualmente através do software Adobe Photoshop a partir dos valores hexadecimais RGB, a fim de facilitar a compreensão da composição da cor no sistema subtrativo, universo mais familiar para designers gráficos. Além disso foi acrescido mais um item, a tinta metálica dourada, presente nas amostras, que ganhou o número 268.

Figura 8 - Amostra da tabela de referência para identificação de cores. Linguagem de Cor Universal, valores no Sistema de cor de Munsell, conversões em RGB e CMYK acompanhados de amostra visual.

\begin{tabular}{|c|c|c|c|c|c|c|}
\hline $\mathbf{N}^{\circ}$ & Nomenclatura em Inglês & Tradução para o Português & Munsell & $\begin{array}{l}\text { Hexcode } \\
\text { RGB }\end{array}$ & CMYK & Amostra \\
\hline $\mathbf{P k}$ & Pink & \multicolumn{5}{|l|}{ Rosa } \\
\hline 1 & Vivid Pink & Rosa Vívido & 1R 8.0/13.0 & \#FFB5BA & 040200 & \\
\hline 2 & Strong Pink & Rosa Forte & $1.2 \mathrm{R} 6.9 / 8.2$ & \#EA9399 & 050300 & \\
\hline 3 & Deep Pink & Rosa Profundo & 2.1R 6.0/11.1 & \#E4717A & 070400 & \\
\hline 4 & Light Pink & Rosa Claro & $2.6 \mathrm{R} 8.5 / 4.0$ & \#F9CCCA & 030150 & \\
\hline 5 & Moderate Pink & Rosa Moderado & 2.8R 7.2/5.3 & \#DEA5A4 & 045300 & \\
\hline 6 & Dark Pink & Rosa Escuro & 2.7R $5.9 / 6.1$ & \#C08081 & 1060405 & \\
\hline 7 & Pale Pink & Rosa Pálido & 2.0R 8.7/2.1 & \#EAD8D7 & 520100 & \\
\hline 8 & Grayish Pink & Rosa Acinzentado & 2.6R $7.2 / 2.3$ & \#C4AEAD & 2030255 & \\
\hline 9 & Pinkish White & Branco Rosado & 5.8R 9.0/0.8 & \#EAE3E1 & 1010100 & \\
\hline 10 & Pinkish Gray & Cinza Rosado & 9.8R 7.4/1.0 & \#C1B6B3 & 2525255 & \\
\hline
\end{tabular}

Fonte: a autora a partir de Foster, 2006 (http://tx4.us/nbs-iscc.htm)

\section{Confecção e uso de uma escala de referência cromática}

Figura 9 - Escala de referência cromática produzida para identificação de amostras de cor nos impressos cromolitográficos do corpus de estudo.

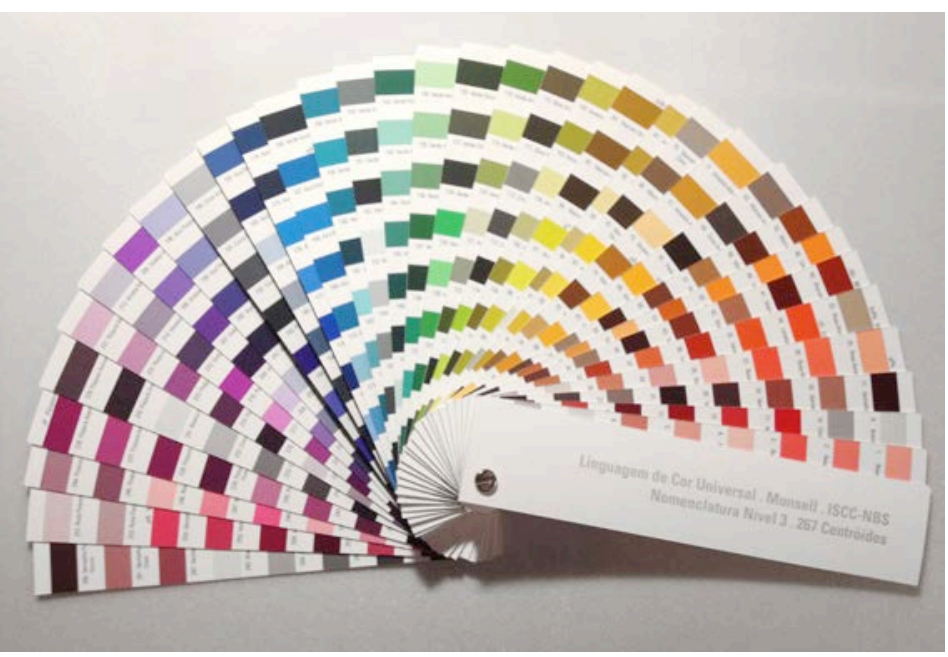

Fonte: a autora.

A partir da referida tabela, foi preparada uma escala de referência cromática, 
ordenada em sequencia numérica para a identificação das cores operantes nas amostras impressas do corpus de estudo da pesquisa. As áreas de cor foram dispostas em retângulos de 4,5 X 1,2cm para facilitar a visualização. Esta escala foi produzida em impressão de arte com tintas pigmentadas, em papel de algodão - a forma mais fidedigna de impressão colorida que se dispões nos dias de hoje (Figura 9). Para facilitar a consulta, as lâminas impressas foram unidas por um eixo central aparafusado, à maneira de uma escala Pantone.

Como comenta Twyman (2013), a busca de um sistema descritivo para designar a cor pode induzir uma falsa noção de precisão. De fato, são muitos os fatores adversos:

a) Impressos históricos estão sujeitos às ações do tempo por exposição à luz e à atmosfera - gerando desbotamento, amarelamento, fungos, etc. - dificilmente se apresentam como foram impressos na época. Essa variação pode ser ainda mais severa se tiverem sido usados pigmentos de baixa qualidade e portanto com menor permanência.

b) Áreas muito pequenas de cor (traços, pontos, granidos e chapiscos) quando observadas no microscópio, são de difícil comparação com uma amostra impressa, já que ambos não são observados simultaneamente. Na maior parte das vezes, as tintas operantes podem estar sobreimpressas, fundindo-se umas com outras, o que dificulta ainda mais a identificação.

c) As condições de iluminação das salas de consulta das Instituições não são adequadas para a identificação de cor.

Porém, diante da inexistência de provas progressivas ou de outro material que pudesse esclarecer o elenco de tintas operantes adotados nos impressos, a identificação de cor por amostragem, mesmo diante das falhas e dificuldades seria a única maneira de tangenciar a questão. Em função da boa conservação da maior parte das amostras optouse por lidar com o material em suas condições atuais, desconsiderando como pode ter sido sua aparência no passado.

Pela finalidade da tese, os dispositivos de avaliação (escala de referência cromática, lupas e microscópios) tiveram sua entrada autorizada nas salas de consulta das Instituições de guarda (Biblioteca Nacional e Arquivo Nacional). A identificação foi processada nos 100 rótulos integrantes da pesquisa, tarefa que exigiu alguns meses de trabalho. A designação de cor foi efetuada por comparação visual (Figura 10) entre a observação microscópica (a, ampliada 7X) ou com lupas (c, ampliada 3X) dos rótulos e a escala de referencia cromática (b, escala real), buscando os mínimos vestígios fora de registro que evidenciassem a presença das tintas operantes. O conhecimento de teoria da cor foi utilizado para identificar o produto da sobreposições de tintas, decompondo seus componentes (d). Vale a ressalva que este tipo de abordagem só foi possível pela característica das técnicas gráficas presentes nos rótulos amostrados. Um acervo litográfico baseado exclusivamente na técnica do crayon em pedra áspera, por exemplo, apresentaria partículas tão pequenas e misturadas que inviabilizariam a aplicação deste método. 
Figura 10 - Método de identificação de cor por comparação visual entre a observação no microscópio (a), com lupa (c) e com a escala de referencia cromática (b). O conhecimento de teoria da cor foi utilizado para a dedução dos componentes nas tintas sobrepostas (d).

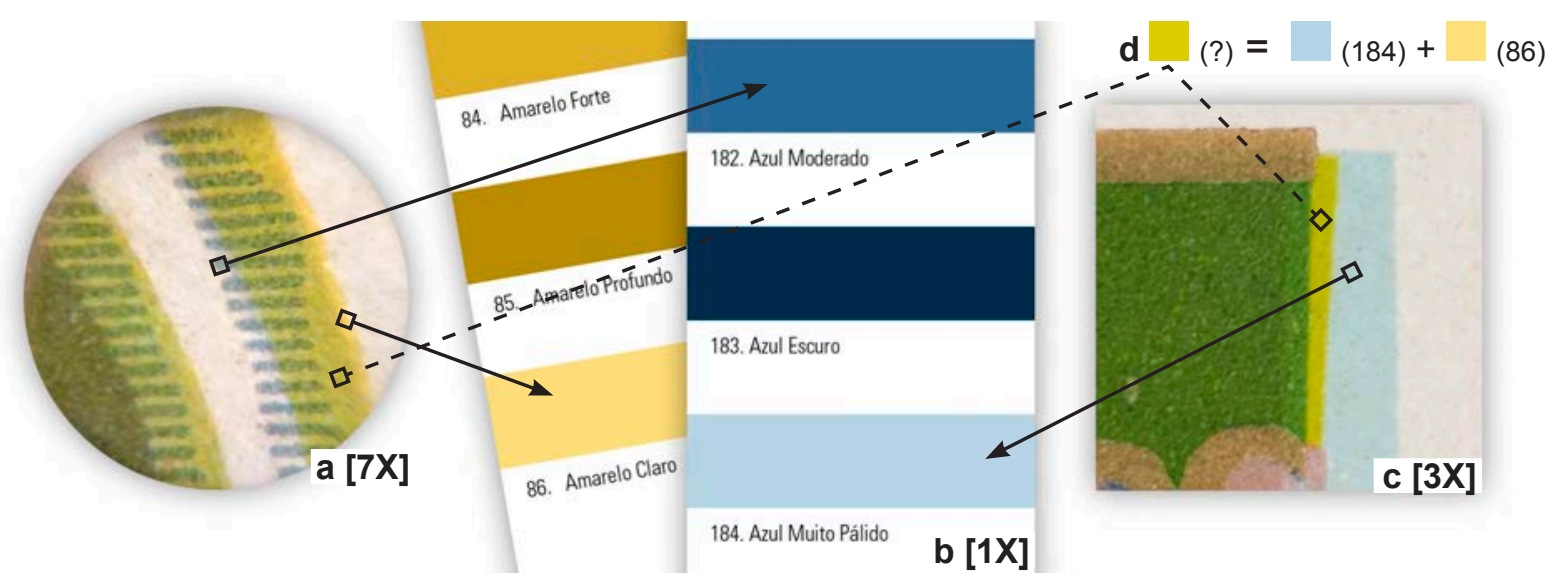

Fonte: a autora

\section{Conclusão}

A escala de referência cromática associada a observação microscópica demonstrouse um recurso metodológico comparativo eficiente, possibilitando equivalências e diferenciações entre as cores identificadas nas amostras. Todo o esforço foi empreendido para uma avaliação coerente, considerando-se as limitações já observadas. Tem-se consciência da falta de rigor técnico, designando, portanto, apenas uma indicação de ordem aproximativa. Tal aproximação, mesmo que imprecisa, remonta as bases de uma prática histórica subjetiva e empírica, quase perdida no tempo. Falhas referem-se possivelmente à presença de mais tintas nos impressos elaborados com mais de 8 cores de seleção, devido a dificuldade de diferenciação diante das muitas sobreposições. Nos impressos com menos tintas, acredita-se que uma boa aproximação foi alcançada.

Voltando-se ao exemplo inicial (Figura 1), se aplicado o método de identificação das tintas operantes pela escala de referência cromática, temos a substituição dos nomes genéricos (rosa, vermelho, argila, amarelo, cinza, azul 1, azul2, profundidade e dourado) por nomes descritivos, não fantasiosos, mapeados por um sistema lógico e compreensivo:

Figura 11 - Identificação de tintas operantes do Rótulo Superior Aguardente Portuguesa Bagaceira (BN), de acordo com a escala de referência cromática.

\begin{tabular}{|l|l|l}
\hline 5 Rosa Moderado & 86 Amarelo Claro & 182 Azul Moderado \\
\hline 12 Vermelho Vívido & 93 Amarelo Acinzentado & 267 Preto \\
\hline 31 Rosa Amarelado Pálido & 184 Azul Muito Pálido & D 268 Dourado
\end{tabular}

Fonte: a autora

Acredita-se que o sistema de nomenclatura de cor aqui proposto pode ser útil não apenas para a pesquisa histórica de impressos, mas a todos os campos do design que desejem se referir as cores fora do contexto de amostras comerciais (como a Escala Pantone, por exemplo), adotando um sistema descritivo e não fantasioso das cores. 
Agradecemos à Capes, ao Arquivo nacional e a Fundação Biblioteca Nacional.

Todos os rótulos apresentados neste artigo foram reproduzidos com autorização da Fundação Biblioteca Nacional e Arquivo Nacional.

\section{Referências}

FOSTER, 2006. Tabela de Nomenclatura de Cor da ISCC-NBS. Disponível em <http://tx4.us/nbs-iscc.htm>, acesso em 10 jan 2016.

JUDD, Deanne B.; KELLY, Kenneth L. Color: Universal Language and dictionary names. Washington: U.S. Dept. of Commerce, National Bureau of Standards, 1976.

JUDD, Deanne B.; KELLY, Kenneth L. Method of designating colors. J. Res. NBS 23, 355-366, 1939.

MARZIO, Peter C. The Democratic Art: Chromolithography 1840-1900, pictures for a 19th-century America. Boston: David R. Godine Publisher, 1979.

MUNSELL, A. H. Atlas of the Munsell Color System. Malden, Mass., Wadsworth, Howland \& Co., inc., Printers, 1915. Original fac-símile disponível em https://archive.org/stream/AtlasMunsellcol00Muns\#page/n0/mode/2up

MUNSELL.COM. Disponível em http://munsell.com>, acesso em 15 jan 2016.

MUNSELL, A. H. A Color Notation. A Measured Color System, Based on the three qualities Hue, Value, and Chroma with illustrative models, charts, and a course of study arranged for teachers. 2nd Edition Revised \& Enlarged. Geo. H. Ellis Co. BOSTON, 1907.

NICKERSON, Dorothy. Standardization of Color Names: The ISCC-NBS Method. United States Department Of Agriculture, 1940.

RIDGWAY, Robert, A Nomenclature of Colors for Naturalists and Compendium of Useful Knowledge for Ornithologists. Boston: Little, Brown and Company, 1886.

RIDGWAY, Robert. Color Standards and Color Nomenclature, with Fifty-three Colored Plates and Eleven Hundred and Fifteen Named Colors. Washington, D. C.: Published by the author, 1912.

SYME, Patrick. Werner's Nomenclature of Colours. Reino Unido, 1821.

TABELA DE NOMENCLATURA DE COR DA ISCC-NBS. Disponível em: http://tx4.us/nbs-iscc.htm. Acesso em 10 de janeiro de 2016.

TWYMAN, Michael. A history of chromolithography: printed colour for all. London: British Library, 2013.

WARHURST, B. W. A colour dictionary giving two hundred names of colours. Specially prepared for stamp collectors, 2nd edition, London: Stanley Gibbons, c. 1908. 